\title{
Culturomics and metagenomics: In understanding of environmental resistome
}

\author{
Monika Nowrotek ${ }^{1}$, Lukasz Jałowiecki ${ }^{1}$, Monika Harnisz ${ }^{2}$, Grażyna Anna Plaza (ه) ${ }^{3}$ \\ 1 Microbiology Unit, Institute for Ecology of Industrial Areas, Kossutha 6 Str., 40-844 Katowice, Poland \\ 2 Department of Environmental Microbiology, Faculty of Environmental Sciences, University of Warmia and Mazury, \\ Prawocheńskiego 1 Str., 10-720 Olsztyn, Poland \\ 3 Silesian University of Technology, Faculty of Organization and Management, Institute of Engineering Production, \\ Roosevelta 26 Str., 41-800 Zabrze, Poland
}

\section{H I G H L I G H T S}

- State of the art of culturomics and metagenomics to study resistome was presented.

- The combination of culturomics and metagenomics approaches was proposed.

- The research directions of antibiotic resistance study has been suggested.

\section{A R T I C L E I N F O}

Article history:

Received 14 November 2018

Revised 17 April 2019

Accepted 25 April 2019

Available online 13 June 2019

\section{Keywords:}

Culturomics

Metagenomics

Antibiotic resistance

Resistome

\section{GRAPHIC A B S T R A C T}

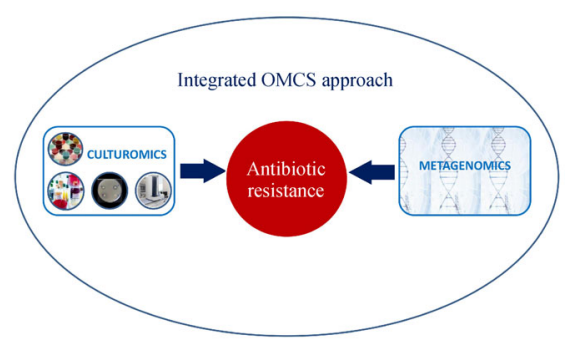

\section{A B S T R A C T}

Pharmaceutical residues, mainly antibiotics, have been called "emerging contaminants" in the environment because of their increasing frequency of detection in aquatic and terrestrial systems and their sublethal ecological effects. Most of them are undiscovered. Both human and veterinary pharmaceuticals, including antibiotics, are introduced into the environment via many different routes, including discharges from municipal wastewater treatment plants and land application of animal manure and biosolids to fertilize croplands. To gain a comprehensive understanding of the widespread problem of antibiotic resistance, modern and scientific approaches have been developed to gain knowledge of the entire antibiotic-resistant microbiota of various ecosystems, which is called the resistome. In this review, two omics methods, i.e. culturomics, a new approach, and metagenomics, used to study antibiotic resistance in environmental samples, are described. Moreover, we discuss how both omics methods have become core scientific tools to characterize microbiomes or resistomes, study natural communities and discover new microbes and new antibiotic resistance genes from environments. The combination of the method for get better outcome of both culturomics and metagenomics will significantly advance our understanding of the role of microbes and their specific properties in the environment.

C The Author(s) 2019. This article is published with open access at link.springer.com and journal.hep. com.cn

\section{Introduction}

For more than a decade, traditional culture - dependent approaches have been the most common methods to study antimicrobial resistance in environmental studies, mainly in wastewater treatment plants (WWTPs), and they have

\section{$\triangle$ Corresponding author}

E-mail: grazyna.plaza@polsl.pl

Special Issue-Environmental Antibiotics and Antibiotic Resistance (Responsible Editors: Xin Yu, Hui Li \& Virender K. Sharma) revealed antibiotic resistance in bacteria over the past years. In these studies, resistance profiles of pathogenic populations of bacterial communities in WWTPs were investigated (Lefkowitz and Duran, 2009; Akiyama and Savin, 2010; Płaza et al., 2013). Culture - dependent methods have also been used to investigate the role of mobile genetic elements (MGEs) in the dissemination of antibiotic resistance genes in WWTPs (Jackson et al., 2011). It has been shown that MGEs influence bacterial evolution and adaptation and play a role in the emergence, recombination, and propagation of antibiotic resistance. Despite the advantages of culture - dependent techniques, 
including low cost and the potential combination with other methods, the availability of culture - based methods for studies of environmental microbes provides a highly restricted view of microbial community structure in environmental samples. The conventional approach of screening for antimicrobial resistance (AMR) involves plating samples on nonselective or antibiotic-selective agar plates, cleaning and isolation of bacterial colonies, and using the standard disc diffusion, broth macrodilution and microdilution or gradient strip methods to determine the MIC (minimal inhibition concentration) values to evaluate resistance to a panel of antibiotics. Routine antibiogram techniques are based on a phenotypic study in which bacterial growth is observed in the presence of different antibiotics. The phenotypic and genotypic characterization methods currently available allow detection of multi-AMR quickly (Anjum, 2015; McLain et al., 2016; Jałowiecki et al., 2017; March-Rosselló, 2017). High-throughput methods increase processing capabilities, data production and analysis of culture-based techniques. Through automated technologies, tests that used to take days can now be performed in a few hours. Isolation of bacteria is a key to understanding and studying phenotypic and genotypic characteristics of individual isolates. However, identification of resistance genes in bulk DNA samples is possible using modern molecular methods. The culture and isolation of individual target pathogens carrying the gene of interest are also essential for determining antibiotic resistance phenotypes. Thus, culture-based studies provide an important link between antibiotic resistance measured in the environment and antibiotic resistance detected in human clinical and environmental isolates (McLain et al., 2016).

However, culture - based methods are not appropriate for comprehensive studies of the diversity and abundance of ARGs as well as the incidence of MGEs in WWTPs (Wang et al., 2013). To date, a variety of molecular approaches have been applied to study AMRs. The application of targeted (PCR - and/or microarray - based) and sequence - based metagenomics provides more extensive and accurate assessments of the abundance of antibiotic resistance bacteria (ARB) and ARGs and the phylogenetic and functional diversity of the environmental resistome.

Previously, both methods were used separately to study AMR. Now, various methods have been applied to study the origin and dissemination of ARB and ARGs, including isolation and culture, PCR, quantitative PCR (qPCR), DNA microarray and metagenomic approaches (Chistoserdova, 2010; Schmieder and Edwards, 2012; Mullany, 2014; Allan, 2014; Anjum, 2015; Pärnänen et al., 2016; Fitzpatrick and Walsh, 2016).

Both molecular and cultivation methods are needed for full insight into resistomes. To date, both methods have been used to study the human gut microbiome (Lagier et al., 2012). Unexpectedly, the authors found divergence between the results obtained by the culture methods (culturomics) and metagenomics at the level of both species and genera. The numerous bacteria detected by culturomics were undetected in metagenomic studies by pyrosequencing (Lagier et al., 2012). Culturomics, coupled with a taxonogenomic strategy, has aided in the mapping of the microbial diversity of the human gut. Masucci et al. (2017) described culturomics as an approach of culture conditions, originally applied to study living bacterial populations considered as unculturable. Most importantly, culturomics was introduced to optimize culture conditions and to show that the term "uncultivable organism" is misleading, since all microorganisms are cultivable using the right conditions and tools (Bilen et al., 2018). The complementarity between culture-independent and culture-dependent studies has been well established in taxonomic studies of human-associated bacterial species. Bilen et al. (2018) report the contribution of culturomics as a strategy complementing metagenomics in describing the taxonomy of human bacterial and archaeal species.

In this review, two complementary tools for studying antibiotic resistance, i.e. culturomics, a new approach of a culture-based omics and metagenomics, are presented.

\section{Culturomics- culture-dependent approach}

\subsection{Culture - based approaches in microbiology}

Traditional methods are based on the ability of the microorganisms to grow under artificial conditions. Some examples are microbiological culture using selective or differential media, microscopy, Gram-staining and biochemical tests. These methods are highly sensitive, reliable, low-priced and provide both qualitative and quantitative results on the bacterial populations present in clinical samples. However, these samples commonly are mixed cultures including diverse pathogens or environmental microorganisms. Typically, the isolation of pure cultures applying several growth steps is necessary to characterize the bacteria in more detail. Consequently, the conventional methods are labor-intensive and time-consuming, since the results are not available for at least 1-3 days. Moreover, the culture-based methods reach their limits with specific microorganisms that do not grow on or in artificial media. In spite of these inconveniences, culture-dependent methods have been utilized for the identification and biochemical characterization of bacterial strains over the past few decades, and recently, the role of culture methods has increased significantly in clinical microbiology.

2.2 Culturomics - the next generation of the culture-based methods

Based on these methods, a new approach known as 
culturomics has been developed. Culturomics was first introduced in 2012 by Prof. Raoult's research group to study human gut microbiota with taxonomic approaches (Lagier et al., 2012; Lagier et al., 2015). Kambouris et al. (2017) declared culturomics as the new kid on the block of omics and described its relationship to the other omics approaches. In Fig. 1, the history of culturomics in clinical microbiology and future potential applications in environmental microbiology are presented. The technique was developed to address shortcomings of metagenomics, which studies genetic material recovered from natural samples. Metagenomics has revolutionized microbial ecology research, but its limitation was the yield of a large number of sequences that could not be assigned to known microorganisms. According to the opinion of many scientists, a growing problem is the gaps in metagenomics approaches, which correspond to unidentified sequences that may be correlated with an identified microbe. In Table 1, some characteristics, limitations and advantages of metagenomics and culturomics are presented. Microbial culturomics has emerged as a successful tool to isolate high numbers of bacteria and to identify new species mainly in the human microbiome (Lagier et al., 2012; Lagier et al., 2015; Lagier et al., 2016; Abdallah et al., 2017; Amrane and Lagier, 2018). It is a new field that complements molecular techniques and gives another approach for determining the composition of microbial populations living in humans and the environment, including Archaea and bacteria. To date, its taxonomic approaches have been adopted by the clinical microbiology field.

\subsection{MALDI - TOF as a useful tools for identification of bacterial colony}

The aim of culturomics is to detect and characterize a broad spectrum of bacteria per sample. Serial dilutions of one sample are distributed on agar media with different culture conditions, and after growth, each bacterial colony is cleaned before identification by Matrix Assisted Laser Desorption Ionisation-Time of Flight (MALDI-TOF) mass spectrometry, providing its taxonomic identification or $16 \mathrm{~S}$ rRNA amplification and sequencing if identification by MALDI-TOF is not possible. Culturomics is defined by Greub (2012) as an approach allowing an extensive assessment of microbial composition by high-throughput culture. This strategy, based on the diversification of culture conditions, has enabled the isolation of more than 1000 distinct human-associated bacterial species since 2012, including 247 new species. The workflow of a culturomics study is presented in Fig. 2. Many different bacterial colonies were identified using the MALDI-TOF coupled to smart incubators and automated colony-picking systems to constitute the next generation of culturomic approaches. Rapid identification using mass spectrometry tools (MALDI-TOF) has been developed recently, and confirmation has been provided by molecular techniques that a number of species were unknown.

\subsection{Application of culturomics approaches - the examples from literature}

Lagier et al. (2012) have identified approximately 32500 different colonies isolated from human stools. They applied 212 different culture conditions, e.g., incubation temperatures, various oxygen conditions, and large variety of enrichment broths and media, to successfully isolate 340 different bacterial species. Among them, 32 new species, representing approximately one-third of species recovered by culture, have been discovered. Interestingly, Lagier et al. (2012) also used metagenomic analysis on the

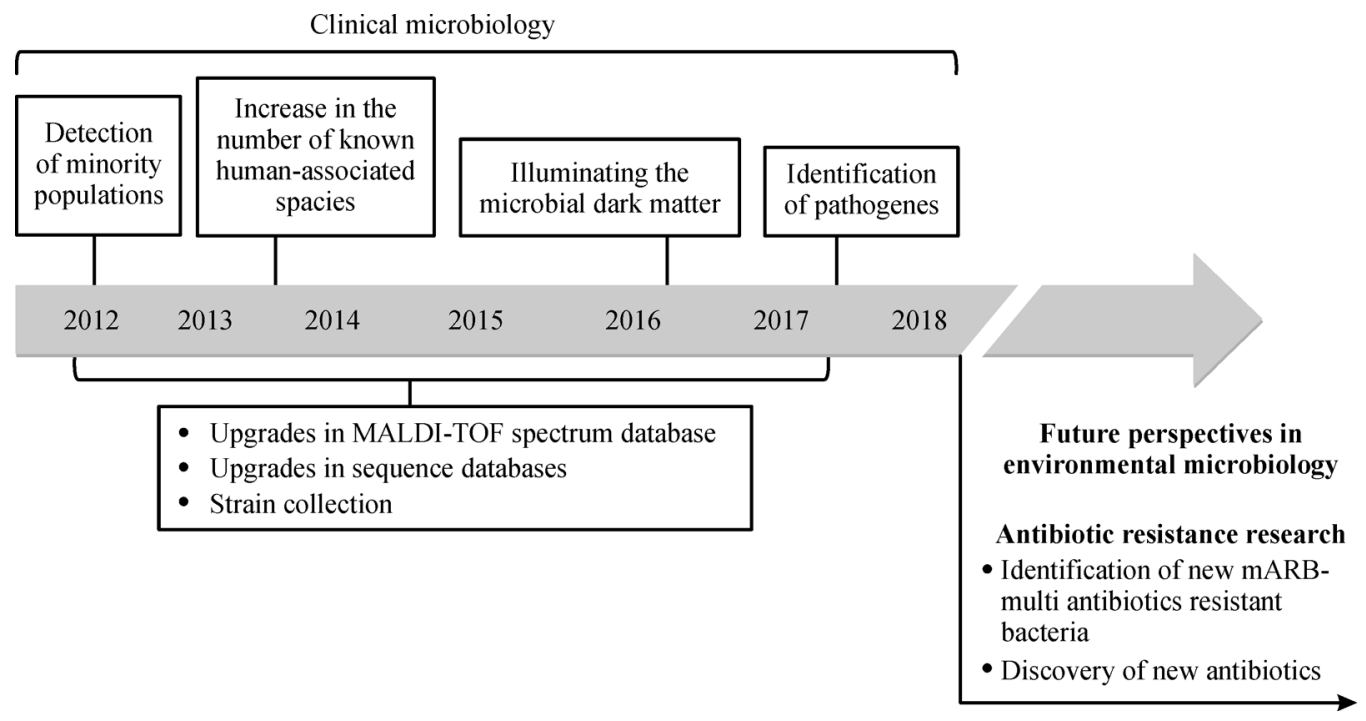

Fig. 1 The history of culturomics approach in clinical microbiology and possible its applications in environmental microbiology (adopted from Lagier et al. (2018)). 
Table 1 Comparison between metagenomics and culturomics (according to Greub (2012))

\begin{tabular}{|c|c|c|}
\hline Features & Metagenomics & Culturomics \\
\hline Definition & $\begin{array}{l}\text { Method allowing the description of microbial composition } \\
\text { by high-throughput sequencing }\end{array}$ & $\begin{array}{l}\text { Method allowing the description of the microbial composition by } \\
\text { high-throughput culture }\end{array}$ \\
\hline Methodology & Pyrosequencing of $16 \mathrm{~S}$ rRNA amplicons & $\begin{array}{l}\text { Use of various selective and/or enrichment culture conditions coupled } \\
\text { to MALDI-TOF MS identification }\end{array}$ \\
\hline Limitations & $\begin{array}{l}\text { Does not provide a strain for further studies. } \\
\text { Not complete population (depth bias) }{ }^{\mathrm{a}} \text {. } \\
\text { Only detects eubacteria. } \\
\text { Does not provide information on enzymatic abilities and } \\
\text { specific metabolites }{ }^{\mathrm{b}} \text {. } \\
\text { Detects "non-cultivable" microbes }\end{array}$ & $\begin{array}{l}\text { Misses so-called "non-cultivable" microbes. } \\
\text { Does not directly provide information on enzymatic abilities. } \\
\text { Major workload }\end{array}$ \\
\hline Advantages & Detects "non-cultivable" microbes & $\begin{array}{l}\text { Detect not complete populations. } \\
\text { Open approach. } \\
\text { Detects only viable bacteria }^{c}\end{array}$ \\
\hline Rate of success & Approximately 200 bacterial species/sample ${ }^{d}$ & Approximately 100 bacterial species/sample ${ }^{d}$ \\
\hline $\begin{array}{l}\text { Possible future } \\
\text { development }\end{array}$ & $\begin{array}{l}\text { Increased deph of sequencing because of new technology. } \\
\text { Coupling pyroseqencing with direct metagenomics }\end{array}$ & $\begin{array}{l}\text { Automated detection of microbial growth } \\
\text { Automated identification } \\
\text { f. Miniaturization. } \\
\text { Other innovative culture conditions }\end{array}$ \\
\hline
\end{tabular}

Notes: a - limitation of direct metagenomics (no amplification step); $\mathrm{b}$ - limitation of pyrosequencing of $16 \mathrm{~S}$ rRNA amplicons; $\mathrm{c}-$ relevance of dead microorganisms lower than that of viable microorganisms (no metabolic activity); $d$ - mean number of bacterial species recovered from one stool sample by Lagier et al. (2012) when comparing both approaches; e - smart incubators, $\mathrm{pH}$ indicators in broth, microcalorimetry, 96 -wells microplates, multireader, etc.; f - automated colony picking coupled to MALDI-TOF MS and/or full laboratory automation.

Steps

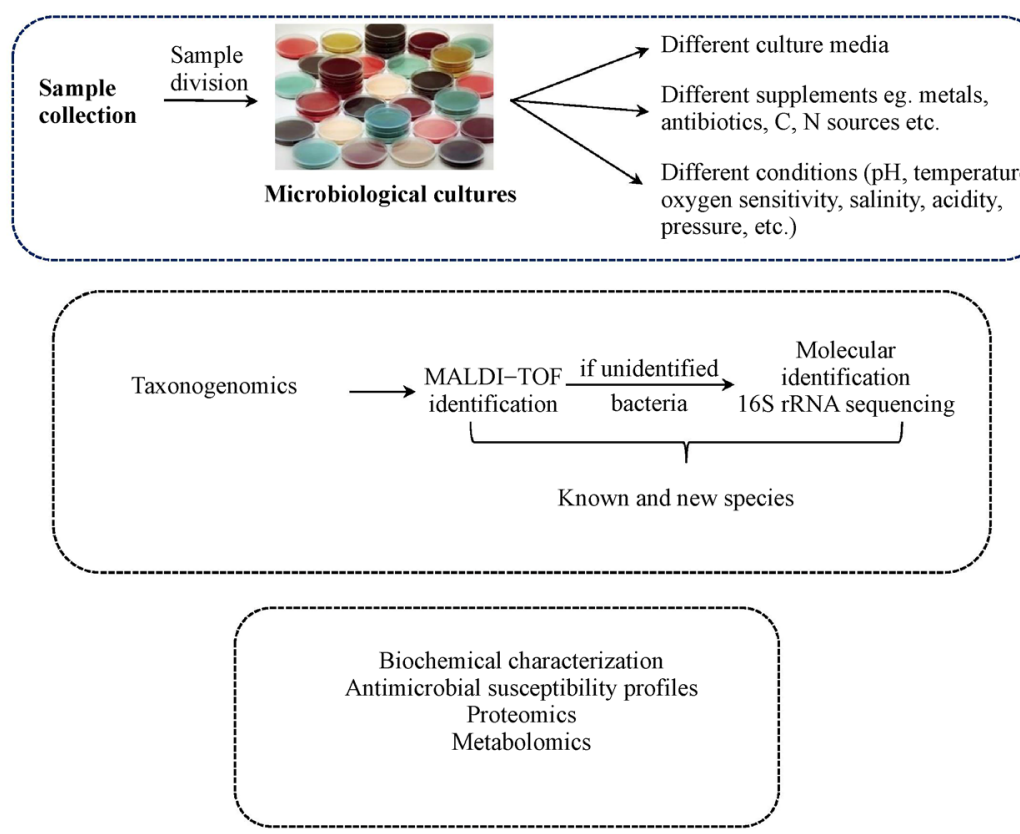

Results
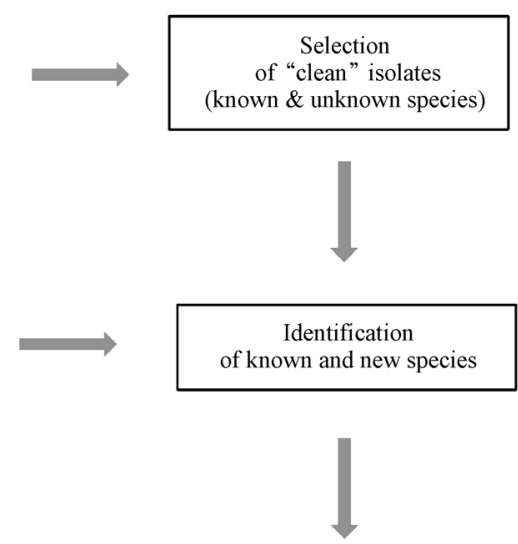

Characterization of known and new species

Fig. 2 The methodology of culturomics approach.

same human samples by pyrosequencing $16 \mathrm{~S}$ rRNA amplicons, and only 51 species identified were among the 340 cultured species. The research demonstrated that culturomics appeared to be an ideal complementary approach to metagenomics in antibiotic resistance studies. In the future, culturomics will be one major approach for studying the human and environmental microbiome, in addition to metagenomics. Microbial culturomics is a field that complements molecular techniques and provides another view of the composition and characterization of microbial populations living in humans and environments. In culturomics research, various culture conditions are applied: various broth and solid media, blood culture bottles, selective agar media, different temperatures and times of incubation from a few days to months, various $\mathrm{pH}$, oxygen and pressure sensitivity. 
To date, culturomics analysis has been used in taxonomic identification of clinical microbes and in the discovery of new species (Khelaifia et al., 2016; Masucci et al., 2017; Bilen et al., 2018; Seck et al., 2018). Khelaifia et al. (2016) reported the description of a new bacterial species using culturomics. The strain was isolated from the stool specimen of a patient from Saudi Arabia. The phenotypic characteristics of the bacteria and its complete genome sequence and annotation were determined. Based on the presented characteristics and identification, the authors proposed classifying this novel halophilic bacterium as representative of a new species belonging to the genus Oceanobacillus, Oceanobacillus jeddahense sp. nov. In the study of Masucci et al. (2017), culturomics was used for isolation of Clostridium difficile from faecal samples obtained from healthy donors of faecal microbiota transplantation (FMT). Further, Seck et al. (2018) used a culturomics study to isolate the halophilic bacterium Bacillus saliss sp. nov. from table salt using high-salt conditions to cultivate halophilic bacteria from environmental samples.

The review described by Bilen et al. (2018) updated the inventory of prokaryotes that have been isolated from different sites on the human body and complements the work published by Hugon et al. (2015), who reported 2172 different species as pathogens or commensals. Additionally, it aims to highlight the contribution of culturomics in describing the human microbiota. Culturomics was presented as a tool proven to be essential in describing new prokaryotic species and filling metagenomic gaps. As described, culturomics contributed up to $66.2 \%$ of the updates to this repertoire by reporting 400 species, of which 288 were novel. The authors of the review demonstrated the need to perform the culturing work, which seemed essential to decipher not only the hidden human microbial content but also the environmental microbial content. The human microbiome is a diverse reservoir of antibiotic resistance genes and the culturomics approach will be a useful tool to identify new antibioticresistant bacteria and new antibiotic resistance. McLain et al. (2016) discussed the use of culture-based methods for detection of antibiotic resistance in agroecosystems. This review covers current knowledge on using culturomics and metagenomics in assessment of antibiotic resistance. The common tests, e.g., broth dilution, agar disc diffusion, agar breakpoints and E-tests, used in culture-based methodology for evaluation of antibiotic resistance are described in this review. The culture and isolation of individual microbes carrying antibiotic resistance genes, in particular, are essential for determining multi-antibiotic resistance phenotypes.

\subsection{Culturomics - the summary}

Microbial culturomics is a new approach in the biological exploration strategies of the 21 st century and could be involved in antibiotic resistance research. By multiplying the isolation strategies and quickly identifying by MALDITOF techniques or sequencing the 16S rDNA gene of bacteria that are not identified in the MALDI-TOF databasenew bacteria with specific properties will be isolated. The results obtained from culturomics show that it has a performance comparable to that of metagenomics with only $15 \%$ of bacteria found in common by metagenomics and culturomics (Bilen et al., 2018). Culturomics will benefit from further progress in omics technologies, e.g. continued decrease in costs for sequencing as a driver for further progress in omics fields and the availability of cultures enables genome sequencing and many possible biotechnological applications. In Fig. 3, the number of articles published on culturomics as a function of time is presented.

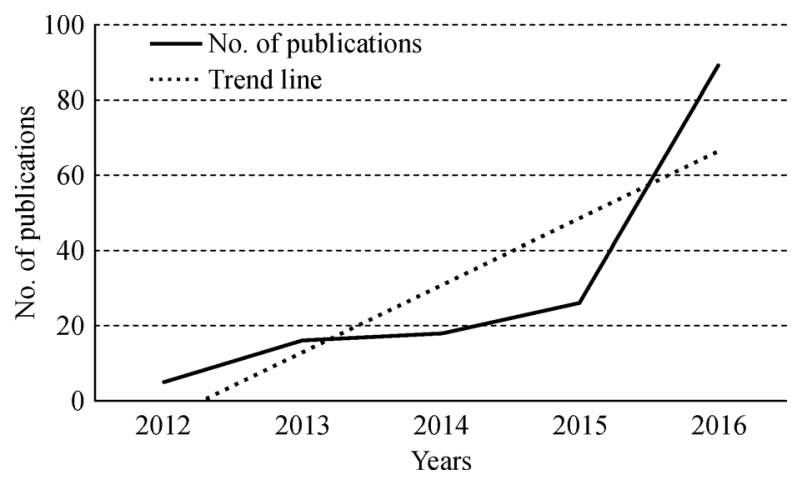

Fig. 3 Number of articles published on culturomics in the past 4 years (Kambouris et al., 2017).

\section{Different examples of molecular approaches}

In modern microbiota studies, there has been a trend toward displacing traditional microbiology methods with genomics approaches and development of culture-independent methods. This trend was based on the concept of uncultivable microbiota. It was estimated that $99 \%$ or more of environmental bacteria are not readily cultured using standard methods (Luby et al., 2016).

The culture - independent methods used to identify the diversity and activity of microorganisms in environmental samples are divided into two groups: one group is partial community analysis approaches, including denaturing gradient gel electrophoresis (DGGE), temperature gradient gel electrophoresis (TGGE), single strand conformation polymorphism (SSCP), DNA (DNA) microarrays, real time - polymerase chain reaction (RT - PCR), and fluorescence in situ hybridization (FISH), and the second one is whole community analysis approaches, including whole genome sequencing, sequenced-based metagenomics, $\mathrm{G}+\mathrm{C}$ fractionation, functional metagenomics, metatranscriptomics and metaproteomics (Mohammadali 
and Devies, 2018). The review of Luby et al. (2016) provides an overview of existing molecular methods for tracking antibiotic resistance in ecosystems, defines their strengths and weaknesses, and recommends their future applications in the characterization of various microbiota. The ongoing development of metagenomic approaches explores antibiotic resistance in the environment and asks the questions that could not be answered by conventional culture-based strategies.

\section{Definition and history of metagenomic approaches}

Metagenomic approaches have recently become the best way to gain comprehensive knowledge of the microbial communities of interest. The term metagenome was proposed by Handelsman et al. (1998) to describe "the genomes of the total microbiota found in nature." Correspondingly, metagenomics is defined as the cultureindependent genomic analysis of microbial communities based on DNA isolated directly from environmental samples.

The direct cloning of environmental DNA was proposed by Pace et al. in 1985. However, the first successful creation of metagenomic libraries was performed by the authors in 1991 (Schmidt et al., 1991). The first metagenomic library was generated by DNA isolated from marine picoplankton. Initially, in metagenomic experiments, total genomic DNA was isolated from the environment, randomly fragmented and sequenced. This analysis allowed the researcher to identify the genes possessed and the metabolic processes performed by those microbial communities, including unculturable organisms in the environment. The development of next-generation sequencing techniques led to sequencing total DNA directly from the samples. This approach was sometimes called random community genomics, which also became known as metagenomics. Metagenomic sequencing was an alternative to rRNA sequencing for evaluation of diversity of microbial communities in environmental samples. One of the most illustrative examples of how metagenomics approaches are a feasible way to accumulate genomic knowledge is the "shotgun" sequencing of the Sargasso Sea waters performed by Venter's research group in 2004 . In this study, almost $1.5 \mathrm{Gbp}$ of microbial sequences from three marine sites was recovered. This led to the finding of almost 70 thousand novel genes (Venter et al., 2004). In the review of de Fatima Alves et al. (2018), the genes discovered through metagenomic approaches with high biotechnological potential are presented.

Currently, metagenomics studies can be divided into different approaches: functional metagenomics and sequence-based metagenomics, and they are becoming increasingly popular in large-scale genomics applications as a way to study the taxonomic and functional composi- tion of microbial communities from environmental, agricultural, and clinical samples.

They have been developed because it is estimated in the literature that less than $1 \%$ of the microorganisms present in different environments are culturable, and our current knowledge of the structural and functional diversity of microbiota is limited. Metagenomics tries to answer some biological questions: "Who are the members of the community?" and "What are their functional roles?". In Table 2, the major stages in metagenomics development over the past 40 years, since Sanger sequencing, and over the past 30 years, since the first published metagenomic experiment, are presented.

Although the concept of "metagenome" and "metagenomics" had been known for nearly 20 years, the application of metagenomics has flourished in recent years owing to the rapid advancement and the decreased cost of high throughput sequencing technology. How many genes or strains could be discovered in metagenomic sequences is largely dependent on the depth of sequencing and the complexity of the community. Multiple sequencing platforms have been developed (e.g. Illumina, 454, SOLiD, and Ion Torrent), and the cost of high throughput sequencing can drop to $\$ 0.02$ per million bases using the Illumina Hiseq 2000 platform, the throughput of which can approach $600 \mathrm{G}$ bases in one run (Di Bella et al., 2013). Using metagenomics, several novel antibiotic resistance genes have been identified, including $\beta$-lactams, tetracycline, aminoglycoside and bleomycin (Schmieder and Edwards, 2012).

\subsection{Types of metagenomic approaches}

Three different metagenomic approaches have been described by Mullany (2014) to investigate the microbiome/resistome: (1) targeted (PCR- and/or microarraybased), (2) sequenced-based, and (3) functional metagenomics. The approaches are able to detect a range of different resistance genes which determine antibiotic resistance in microbiota. In particular, functional metagenomics is a powerful tool for the detection of expression, identification and isolation of novel antibiotic resistance genes from the unculturable fraction of the microbiota. However, the combination of results from both types of metagenomics, e.g., sequenced-based and functional, allows the identification of mobile genetic elements containing antibiotic resistance genes and the correlation of resistance genes with community structure. In Fig. 4, an overview of metagenomic approaches is presented.

Targeted (PCR-based) metagenomics can be used to detect the presence of specific resistance genes or gene families within or between various ecosystems. The following PCRs, including standard, real-time and multiplex PCRs, are now applied to look for the presence of AMR (antimicrobial resistance) genes. PCR primers are designed for the amplification of resistance genes of 
Table 2 Timeline of the major stages in metagenomics research (modified from Escobar-Zepeda et al. (2015); Alves et al. (2018))

\begin{tabular}{|c|c|}
\hline Time & Milestones \\
\hline 1676 & Leeuwenhoek, his observations on oral microbiota \\
\hline 1888 & Koch R., isolation of microbes on solid media \\
\hline 1931 & Winogradsky, microbial ecology experiments \\
\hline 1953 & J.D. Watson and F. Crick published "a radically different structure" for DNA \\
\hline 1977 & Sanger et al. develop DNA sequencing; rRNA was proposed by Woese C. as marker for taxonomy \\
\hline 1980 & Mullis K. develops PCR \\
\hline 1986 & Pace et al. perform cloning DNA directly from the environmental samples \\
\hline 1990 & Giovannoni et al. perform the first microbial community study by $16 \mathrm{~S}$ rRNA libraries \\
\hline 1991 & Schimdt et al. generate metagenomic library from marine plankton \\
\hline 1995 & Healy et al. construct metagenomic libraries from a gene of interest-related environment to mining cellulases \\
\hline 1996 & Stein et al. Describ genomic sequence bearing a $16 \mathrm{~S}$ rRNA gene of an uncultured archaeon \\
\hline 1998 & Handelsman et al. introduce the term "metagenomics" \\
\hline 2004 & Sequencing of the sargasso sea by Venter et al. \\
\hline 2005 & First next-generation sequencing machine released by Roche \\
\hline 2006 & GA sequencer from Solexa is released \\
\hline 2008 & Human microbiome project publication \\
\hline 2010 & MetaHIT consortium releases the human gut microbial gene catalog \\
\hline 2011 & PacBio RS sequencer is released \\
\hline 2016 & MetaSUB consortium is created \\
\hline
\end{tabular}

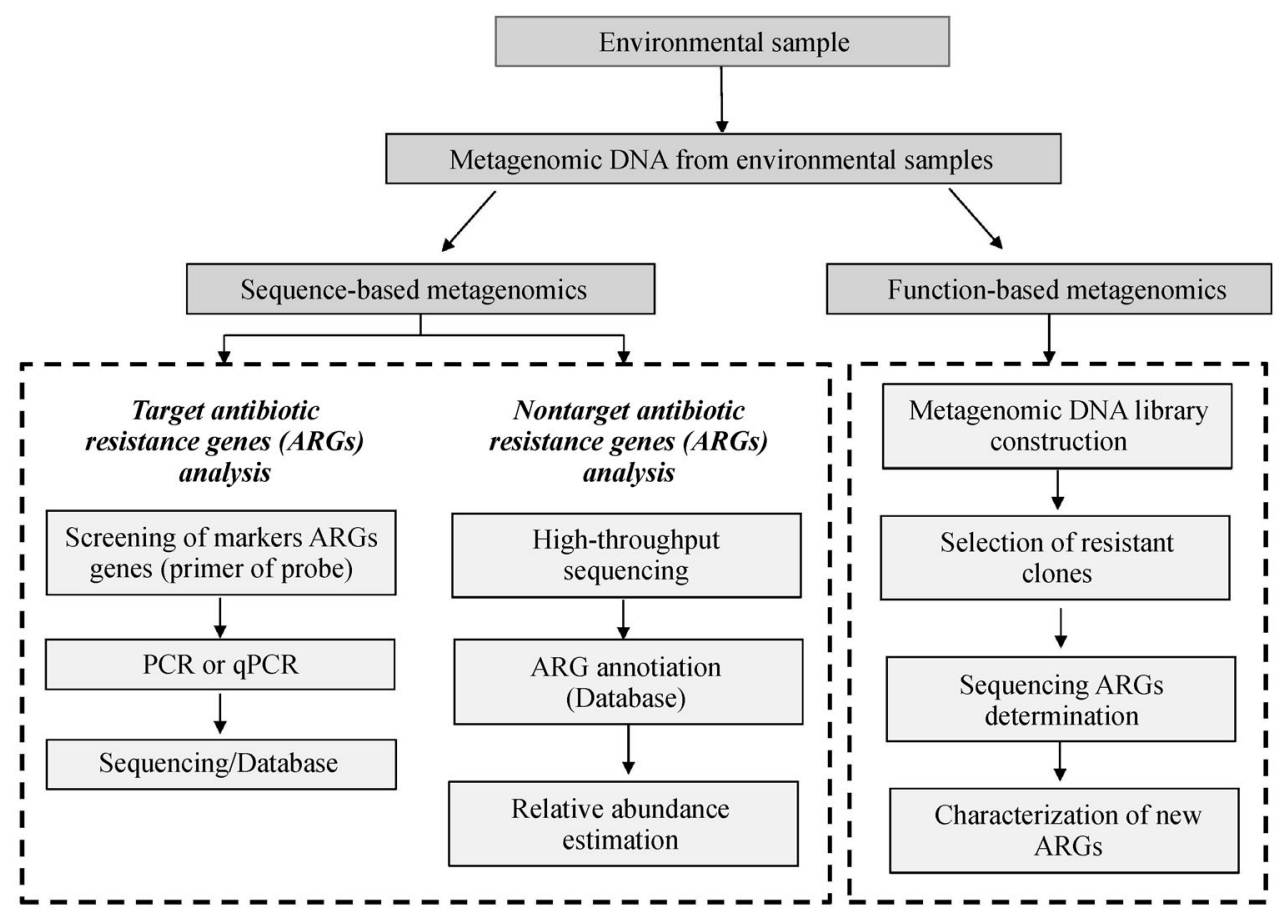

Fig. 4 Overview of metagenomic approaches used in antibiotic resistomes study (adopted from Monier et al. (2011); Schmieder and Edwards (2012)). 
interest within metagenomes. Real-time PCR can be used to produce semiquantitative data to determine the relative abundance of different genes in the microbiome. PCRbased metagenomics allows the detection of all known resistance genes.

In sequenced-based metagenomics, DNA is extracted from the samples, and next generation sequencing technologies are used to obtain complete sequences. The resulting, relatively short contiguous sequence read lengths obtained could then be compared with the public databases to identify resistance genes. This approach allows the identification of all the known and unknown resistance genes in a metagenome. However, this study gives no information on the expression of resistance genes.

\subsection{Metagenomics in environmental studies}

In environmental microbiology, there are two forms of sequenced-based metagenomics. The first is a targeted strategy called deep amplicon sequencing (DAS), which employs a pre-sequencing PCR amplification step. Selective primers for specific and conserved genes, such as $16 \mathrm{~S}$ rRNA, rpoB, and cpn60, are used. The second is a broader strategy known as shotgun metagenomics, in which total DNA isolated from the sample is sequenced directly on a next-generation sequencing platform, and the resulting reads are compared to a reference database. These databases contain all known sequences from many organisms. Using next-generation-based "deep sequencing," many different amplicons in a sample are sequenced. The precise description of the sequencedbased metagenomic approach is presented by Miller et al. (2013).

In the functional metagenomic approach, total DNA is isolated and fragmented with restriction enzymes, and subsequently it is shot-gun ligated into a cloning vector. The vector (plasmids, fosmids, cosmids, or bacteriophages) with the inserted DNA fragment is then cloned into the host. The clones are then plated onto antibiotic containing agar media. Then, DNA is isolated from the clones, and the DNA sequence of the insert is determined (Lam et al., 2015). Mullany (2014) summarizes the features of some of the vector systems used in functional metagenomics to generate gene libraries. According to the studies, the limitation of functional metagenomics is obtaining expression of the resistance genes in the surrogate host (Chistoserdova, 2010; Mullany, 2014). Functional metagenomics has been playing a major role in identification of novel genes, pathways, enzymes, antibiotic resistance genes, and many other bioactive molecules with various biotechnological applications (Alvares et al., 2018).

\subsection{The analysis of ARGs with metagenomics tools}

There are two major challenges in applying metagenomics for studying environmental ARGs: (i) in most natural environments, the relative abundance of ARGs is low, which increases the requirement for sequencing depth and cost substantially, and (ii) the assembly of short reads into longer contigs can be challenging for samples from diverse environments (Pärnänen et al., 2016; Martínez et al., 2017). Recent studies have shown that next generation sequencing technologies have high sequencing depth and accuracy to cover complex bacterial communities and resistomes (Perry et al., 2014; Elbehery et al., 2016; Hamad et al., 2017; Crofts et al., 2017; Lanza et al., 2018). However, both methods have some limitations, e.g. function-based metagenomics limitations are: the screening system is limited to the range of functional activities, not all genes are efficiently expressed in standard hosts and there is a size limitation of metagenomic libraries. Sequencing-based metagenomics, an alternative to functional metagenomics, depends on existing gene annotations, which limits the potential to identify fundamentally novel genes encoding specific activities. Nevertheless, the accumulation of large new data sets in currently available sequencing platforms and development of bioinformatic tools promotes the use of sequencing-based metagenomics as the method for the search of new functional activities, including the genetic determinants of antimicrobial resistance (AMR).

\subsection{Use of metagenomics approaches in evaluation of antibiotic resistance in WWTPs: an example}

Previously, metagenomics approaches have been widely used in the investigation of ARGs in samples isolated from various environments (Chen et al., 2013; Yang et al., 2013; Amos et al., 2014; Christgen et al., 2015; Ma et al., 2016; Xiao et al., 2016). Recently, most studies deal with antibiotic resistance in wastewater treatment plants and receiving rivers (Tang et al., 2016; $\mathrm{Hu}$ et al., 2016; Guo et al., 2017; Lee et al., 2017; Karkman et al., 2017; Rosso et al., 2018; Chu et al., 2018; Gupta et al., 2018). Wastewater treatment plants (WWTPs) are the environments where pathogens, opportunistic pathogens, and environmental bacteria are mixed. The resistance markers are able to spread through the microbial communities and as a result, antibiotic-resistant bacteria can potentially disseminate their resistance genes widely among members of the endogenous microbial community. Effluents from urban wastewater treatment plants (WWTPs) are suspected to be among the main anthropogenic sources for antibiotics, ARB and ARGs spread into the environment (Rizzo et al. 2013). The biological treatment process creates an environment potentially suitable for resistance development and spread because bacteria are continuously mixed with antibiotics at inhibitory concentrations. The treatment used in WWTPs is not efficient enough to remove both $\mathrm{ARB}$ and $\mathrm{ARG}$ that are released to the receiving river (Rizzo et al., 2013). In addition, WWTPs 
link human activities and the environment and may facilitate horizontal transfer of resistance determinants among a rich diversity of commensals, environmental microorganisms and clinically relevant pathogens. In this regard, WWTP may contribute to the occurrence, spread and persistence of both antibiotic-resistant bacteria and antibiotic resistance determinants in the environment.

$\mathrm{Hu}$ et al. (2016) used high-throughput sequencing-based metagenomic approaches to investigate the effect of ultraviolet light on the microbial community structure and diversity and abundance of ARGs in biologically treated wastewater. However, Tang et al. (2016) investigated the changes of the bacterial community and ARGs along treatment processes of one WWTP and examined the effects of the effluent discharge on the bacterial community composition and the ARGs diversity in the receiving water. The comparison between the wastewater treatment process and the receiving river revealed the overall profiles of bacterial communities and ARGs responding to the discharge of treated effluent in the receiving river. Their results showed slightly higher abundance of potential pathogens and ARGs in downstream water samples than in the upstream samples, indicating that the effluent from WWTP might induce possible health risks of the receiving river water in terms of bacterial pathogenesis and antibiotic resistance. Additionally, Wang et al. (2013) used Illumina high-throughput sequencing to comprehensively investigate the microbial community structure and function of anaerobic and aerobic sludge in a full-scale tannery WWTP, with emphasis on abundance and diversity of ARGs and MGEs in the sludge. Illumnia high-throughput sequencing was used to investigate the broad-spectrum profiles (occurrence, abundance and diversity) of antibiotic resistance genes and mobile genetic elements in activated sludge collected from WWTP by Guo et al. (2017). The article of Rosso et al. (2018) presented the use of shotgun metagenomics to characterize populations of a foamy activated sludge aeration basin, which promotes aerobic respiration of carbon and nitrogen compounds. This work showed that foam production is associated with blooms of mycolic acid-producing genera, especially Mycobacterium, and the samples had unusual nitrifying populations. Chu et al. (2018) also used shotgun metagenomics for the characterization of antibiotic genes and organisms (genetic and taxonomic context) from two WWTPs and lake sediments close to the effluent discharge. Their study allowed the specific link of genes to organisms and their genetic context, providing information on impacts of WWTPs on natural microbial communities. The obtained results suggest the influence of wastewater effluents on antibiotic gene content and microbial community structure in the sediments of receiving lake waters. They tracked the genetic footprint, including antibiotic resistance genes, mobile genetic elements and associated microbes, from WWTPs to lake sediments. It was found that many ARGs were located on mobile genetic elements in both the effluent and sediment samples, indicating horizontal gene transfer as the main mechanism of ARG movement. The linking of taxa and their genes within the communities and determining their relationship with their location was accomplished using a metagenomic approach. In another paper, high-throughput sequencing using the Illumnia HiSeq 4000 platform was used to study antibiotic resistance genes (ARGs), heavy metal resistance genes (HMRGs), as well as bacterial diversity and mobile genetic elements, in influent and effluent at the WWPT in South Korea (Gupta et al., 2018). The study demonstrates that many ARGs and HMRGs are not sensitive to treatment processes in WWTPs, and their association to MGEs may contribute to the movement of resistance genes among environmental microbes.

Further, Gatica et al. (2016) present a comprehensive overview of gene cassette composition in effluents from wastewater treatment facilities across Europe. High levels of antibiotic resistance genes have been found in the river sediments downstream from the wastewater treatment plants using sequence-based metagenomics. The resistance genes made up almost $2 \%$ of the DNA samples taken.

\section{Summary and recommendations}

Establishing the optimal methodology to quantitatively and accurately assess the antimicrobial sensitivity of environmental microbes is essential. This importance was realized through the advent of-omics technologies and their application to improve our knowledge of environmental microbiomes. In particular, the use of metagenomics has revealed the diversity of environmental microbiota, but it has also highlighted that the majority of bacteria are uncultured. However, culturomics develops culture methods to identify unknown bacteria as a part of the rebirth of culture techniques. Consisting of multiple culture conditions combined with the rapid identification of bacteria, the culturomics approach has enabled the culture of hundreds of new microorganisms with various and specific properties.

In this review, both complementary methodologies, culturomics and metagenomics, are presented. We describe how culturomics has extended our understanding of bacterial diversity and then explore how culturomics can be applied to the study of the environmental microbiota. The combination of both culturomics and metagenomics approaches will significantly advance our understanding of the role of microbes and their specific properties. The current methodology based on-omics methods improves resistome assessment in the environment.

Acknowledgements This paper was prepared in connection with the work done under the project No. 2017/26/M/NZ9/00071 funded by the National Science Center (Poland). 
Ethical approval This article does not contain any studies with human participants or animals performed by any of the authors.

Open Access This article is licensed under a Creative Commons Attribution 4.0 International License, which permits use, sharing, adaptation, distribution and reproduction in any medium or format, as long as you give appropriate credit to the original author(s) and the source, provide a link to the Creative Commons licence, and indicate if changes were made. The images or other third party material in this article are included in the article's Creative Commons licence, unless indicated otherwise in a credit line to the material. If material is not included in the article's Creative Commons licence and your intended use is not permitted by statutory regulation or exceeds the permitted use, you will need to obtain permission directly from the copyright holder. To view a copy of this licence, visit http://creativecommons.org/licenses/by/4.0/.

\section{References}

Abdallah R A, Beye M, Diop A, Bakour S, Raoult D, Fournier P E (2017). The impact of culturomics on taxonomy in clinical microbiology. Antonie van Leeuwenhoek, 110(10): 1327-1337

Akiyama T, Savin M C (2010). Populations of antibiotic-resistant coliform bacteria change rapidly in a wastewater effluent dominated stream. The Science of the total environment, 408(24): 6192-6201

Allan E (2014). Metagenomics: unrestricted access to microbial communities. Virulence, 5(3): 397-398

Alves L F, Westmann C A, Lovate G L, de Siqueira G M V, Borelli T C, Guazzaroni M E (2018). Metagenomic approaches for understanding new concepts in microbial science. International Journal of Genomics, 2018: 1

Amos G C A, Zhang L, Hawkey P M, Gaze W H, Wellington E M (2014). Functional metagenomic analysis reveals rivers are a reservoir for diverse antibiotic resistance genes. Veterinary Microbiology, 171(3-4): 441-447

Amrane S, Lagier J C (2018). Metagenomic and clinical microbiology. Human Microbiome Journal, 9(1): 1-6

Anjum M F (2015). Screening methods for the detection of antimicrobial resistance genes present in bacterial isolates and the microbiota. Future Microbiology, 10(3): 317-320

Bilen M, Dufour J C, Lagier J C, Cadoret F, Daoud Z, Dubourg G, Raoult D (2018). The contribution of culturomics to the repertoire of isolated human bacterial and archaeal species. Microbiome, 6(1): 94

Chen B, Yang Y, Liang X, Yu K, Zhang T, Li X (2013). Metagenomic profiles of antibiotic resistance genes (ARGs) between human impacted estuary and deep ocean sediments. Environmental Science \& Technology, 47(22): 12753-12760

Chistoserdova L (2010). Functional metagenomics: recent advances and future challenges. Biotechnology \& Genetic Engineering Reviews, 26(1): 335-352

Christgen B, Yang Y, Ahammad S Z, Li B, Rodriquez D C, Zhang T, Graham D W (2015). Metagenomics shows that low-energy anaerobic-aerobic treatment reactors reduce antibiotic resistance gene levels from domestic wastewater. Environmental Science \& Technology, 49(4): 2577-2584

Chu B T T, Petrovich M L, Chaudhary A, Wright D, Murphy B, Wells G, Poretsky R (2018). Metagenomics reveals the impact of wastewater treatment plants on the dispersal of microorganisms and genes in aquatic sediments. Applied and Environmental Microbiology, 84(5): e02168-e17

Crofts T S, Gasparrini A J, Dantas G (2017). Next-generation approaches to understand and combat the antibiotic resistome. Nature Reviews. Microbiology, 15(7): 422-434

Davies J, Davies D (2010). Origins and evolution of antibiotic resistance. Microbiology Molecular Reports, 74(3): 417-433

Di Bella J M, Bao Y, Gloor G B, Burton J P, Reid G (2013). High throughput sequencing methods and analysis for microbiome research. Journal of Microbiological Methods, 95(3): 401-414

Elbehery A H A, Aziz R K, Siam R (2016). Antibiotic resistome: Improving detection and quantification accuracy for comparative metagenomics. OMICS: A Journal of Integrative Biology, 20(4): 229-238

Escobar-Zepeda A, Vera-Ponce de Leon A, Sanchez-Flores A (2015). The road to metagenomics: From microbiology to DNA sequencing technologies and bioinformatics. Froniers in Genetics, 6: 348

Fitzpatrick D, Walsh F (2016). Antibiotic resistance genes across a wide variety of metagenomes. FEMS Microbiology Ecology, 92(2): 11-21

Gatica J, Tripathi V, Green S, Manaia C M, Berendonk T, Cacace D, Merlin C, Kreuzinger N, Schwartz T, Fatta-Kassinos D, Rizzo L, Schwermer C U, Garelick H, Jurkevitch E, Cytryn E (2016). High throughput analysis of integrin gene cassettes in wastewater environments. Environmental Science \& Technology, 50(21): 11825-11836

Greub G (2012). Culturomics: A new approach to study the human microbiome. Clinical Microbiology and Infection, 18(12): 11571159

Guo J, Li J, Chen H, Bond P L, Yuan Z (2017). Metagenomic analysis reveals wastewater treatment plants as hotspots of antibiotic resistance genes and mobile genetic elements. Water Research, 123(3): 468-478

Gupta S K, Shin H, Han D, Hur H G, Unno T (2018). Metagenomic analysis reveals the prevalence and persistence of antibiotic- and heavy metal-resistance genes in wastewater treatment plant. Journal of Microbiology (Seoul, Korea), 56(6): 408-415

Hamad I, Ranque S, Azhar E I, Yasir M, Jiman-Fatani A A, TissotDupont H, Raoult D, Bittar F, Bittar F (2017). Culturomics and Amplicon-based Metagenomic Approaches for the Study of Fungal Population in Human Gut Microbiota. Scientific Reports, 7(1): 16788 Handelsman J, Rondon M R, Brady S F, Clardy J, Goodman R M (1998). Molecular biological access to the chemistry of unknown soil microbes: A new frontier for natural products. Chemistry \& Biology, 5(10): R245-R249

Hu Q, Zhang X X, Jia S, Huang K, Tang J, Shi P, Ye L, Ren H (2016). Metagenomic insights into ultraviolet disinfection effects on antibiotic resistome in biologically treated wastewater. Water Research, 101(3): 309-317

Hugon P, Dufour J C, Colson P, Fournier P E, Sallah K, Raoult D (2015). A comprehensive repertoire of prokaryotic species identified in human beings. The Lancet. Infectious Diseases, 15(10): 12111219

Jackson R W, Vinatzer B, Arnold D L, Dorus S, Murillo J (2011). The influence of the accessory genome on bacterial pathogen evolution. Mobile Genetic Elements, 1(1): 55-65

Jałowiecki Ł, Chojniak J, Dorgeloh E, Hegedusova B, Ejhed H, Magnér J, Płaza G (2017). Using phenotype microarrays in the assessment of 
the antibiotic susceptibility profile of bacteria isolated from wastewater in on-site treatment facilities. Folia Microbiologica, 62(6): 453-461

Kambouris M E, Pavlidis C, Skoufas E, Arabatzis M, Kantzanou M, Velegraki A, Patrinos G P (2018). Culturomics: A new kid on the block of OMICS to enable personalized medicine. OMICS: A Journal of Integrative Biology, 22(2), 234-245

Khelaifia S, Lagier J Ch, Bibi F, Azhar E I, Croce O, Padmanabhan R, Jiman-Fatani A A, Yasir M, Robert C, Andrieu C, Fournier P E, Raoult D (2016). Microbial culturomics to map halophilic bacterium in human gut: genome sequence and description of Oceanobacillus jeddahense sp. nov. Journal of Integrative Biolology, 20(4): 248-258

Lagier J C, Armougom F, Million M, Hugon P, Pagnier I, Robert C, Bittar F, Fournous G, Gimenez G, Maraninchi M, Trape J F, Koonin E V, La Scola B, Raoult D (2012). Microbial culturomics: paradigm shift in the human gut microbiome study. Clinical Microbiology and Infection, 18(12): 1185-1193

Lagier J C, Dubourg G, Million M, Cadoret F, Bilen M, Fenollar F, Levasseur A, Rolain J M, Fournier P E, Raoult D (2018). Culturing the human microbiota and culturomics. Nature Reviews. Microbiology, 16(9): 540-550

Lagier J C, Hugon P, Khelaifia S, Fournier P E, La Scola B, Raoult D (2015). The rebirth of culture in microbiology through the example of culturomics to study human gut microbiota. Clinical Microbiology Reviews, 28(1): 237-264

Lagier J C, Khelaifia S, Alou M T, Ndongo S, Dione N, Hugon P, Caputo A, Cadoret F, Traore S I, Seck E H, Dubourg G, Durand G, Mourembou G, Guilhot E, Togo A, Bellali S, Bachar D, Cassir N, Bittar F, Delerce J, Mailhe M, Ricaboni D, Bilen M, Dangui Nieko N P, Dia Badiane N M, Valles C, Mouelhi D, Diop K, Million M, Musso D, Abrahão J, Azhar E I, Bibi F, Yasir M, Diallo A, Sokhna C, Djossou F, Vitton V, Robert C, Rolain J M, La Scola B, Fournier P E, Levasseur A, Raoult D (2016). Culture of previously uncultured members of the human gut microbiota by culturomics. Nature Microbiology, 1(2): 16203

Lam K N, Cheng J, Engel K, Neufeld J D, Charles T C (2015). Current and future resources for functional metagenomics. Frontiers in Microbiology, 6: article1196

Lanza V F, Baquero F, Martínez J L, Ramos-Ruíz R, González-Zorn B, Andremont A, Sánchez-Valenzuela A, Ehrlich S D, Kennedy S, Ruppé E, van Schaik W, Willems R J, de la Cruz F, Coque T M (2018). In-depth resistome analysis by targeted metagenomics. Microbiome, 6(1): 11

Lee J, Jeon J H, Shin J, Jang H M, Kim S, Song M S, Kim Y M (2017). Quantitative and qualitative changes in antibiotic resistance genes after passing through treatment processes in municipal wastewater treatment plants. Science of the Total Environment, 605-606: 906914

Lefkowitz J R, Duran M (2009). Changes in antibiotic resistance patterns of Escherichia coli during domestic wastewater treatment. Water Environment Research, 81(9): 878-885

Luby E, Ibekwe A M, Zilles J, Pruden A (2016). Molecular methods for assessment of antibiotic resistance in agricultural ecosystems: prospects and challenges. Journal of Environmental Quality, 45(2): 441-453

Ma Y, Metch JW, Yang Y, Pruden A, Zhang T (2016). Shift in antibiotic resistance gene profiles associated with nanosilver during wastewater treatment. FEMS Microbiology Ecology, 92(3): pii: fiw022

March-Rosselló G A (2017). Rapid methods for detection of bacterial resistance to antibiotics. Enfermedades Infecciosas y Microbiologia Clinica, 35(3): 182-188

Martínez J L, Coque T M, Lanza V F, de la Cruz F, Baquero F (2017). Genomic and metagenomic technologies to explore the antibiotic resistance mobilome. Annals of the New York Academy of Sciences, 1388(1): 26-41

Masucci L, Quaranta G, Nagel D, Primus S, Romano L, Graffeo R, Ianiro G, Gasbarrini A, Cammarota G, Sanguinetti M (2017). Culturomics: Bacterial species isolated in 3 healthy donors for faecal microbiota transplantation in Clostridium difficile infection. Microbiologia Medica, 32: 6510

McLain J E, Cytryn E, Durso L M, Young S (2016). Culture-based methods for detection of antibiotic resistance in agroecosystems: Advantages, challenges, and gaps in knowledge. Journal of Environmental Quality, 45(2): 432-440

Miller R R, Montoya V, Gardy J L, Patrick D M, Tang P (2013). Metagenomics for pathogen detection in public health. Genome Medicine, 5(9): No article: 81

Mohammadali M, Davies J(2018). Antimicrobial resistance genes and wastewater treatment. In: Keen P L, Fugère R, eds. Antimicrobial Resistance in Wastewater Treatment Processes. 1st ed. Hoboken: John Wiley \& Sons, Inc., 1-14

Monier J M, Demanèche S, Delmont T O, Mathieu A, Vogel T M, Simonet P (2011). Metagenomic exploration of antibiotic resistance in soil. Current Opinion in Microbiology, 14(3): 229-235

Mullany P (2014). Functional metagenomics for the investigation of antibiotic resistance. Virulence, 5(3): 443-447

Nagarajan M. (2018). Metagenomics. Perspectives, Methods, and Applications. 1st ed. London: Academic Press, Elsevier, , 1- 10

Pärnänen K, Karkman A, Tamminen M, Lyra C, Hultman J, Paulin L, Virta M (2016). Evaluating the mobility potential of antibiotic resistance genes in environmental resistomes without metagenomics. Scientific Reports, 6(1): 35790

Perry J A, Westman E L, Wright G D (2014). The antibiotic resistome: What's new? Current Opinion in Microbiology, 21: 45-50

Płaza G, Turek A, Szczygłowska R (2013). Characterization of E. coli strains obtained from wastewater effluent. International Journal of Environmental of Research, 2(1): 67-74

Rizzo L, Manaia C, Merlin C, Schwartz T, Dagot C, Ploy M C, Michael I, Fatta-Kassinos D (2013). Urban wastewater treatment plants as hotspots for antibiotic resistant bacteria and genes spread into the environment: A review. The Science of the total environment, 447: 345-360

Rosso G E, Muday J A, Curran J F (2018). Tools for Metagenomic Analysis at Wastewater Treatment Plants: Application to a Foaming Episode. Water environment research: A research publication of the Water Environment Federation, 90(3): 258-268

Schmidt T M, DeLong E F, Pace N R (1991). Analysis of a marine picoplankton community by $16 \mathrm{~S}$ rRNA gene cloning and sequencing. Journal of Bacteriology, 173(14): 4371-4378

Schmieder R, Edwards R (2012). Insights into antibiotic resistance through metagenomic approaches. Future Microbiology, 7(1): 73-89

Seck E H, Diop A, Armstrong N, Delerce J, Fournier P E, Raoult D, 
Khelaifia S (2018). Microbial culturomics to isolate halophilic bacteria from table salt: genome sequence and description of the moderately halophilic bacterium Bacillus salis sp. nov. New Microbes and New Infections, 23(1): 28-38

Tang J, Bu Y, Zhang X X, Huang K, He X, Ye L, Shan Z, Ren H (2016). Metagenomic analysis of bacterial community composition and antibiotic resistance genes in a wastewater treatment plant and its receiving surface water. Ecotoxicology and Environmental Safety, 132(2): 260-269

Venter J C, Remington K, Heidelberg J F, Halpern A L, Rusch D, Eisen J A, Wu D, Paulsen I, Nelson K E, Nelson W, Fouts D E, Levy S, Knap A H, Lomas M W, Nealson K, White O, Peterson J, Hoffman J, Parsons R, Baden-Tillson H, Pfannkoch C, Rogers Y H, Smith H O
(2004). Environmental genome shotgun sequencing of the Sargasso Sea. Science, 304(5667): 66-74

Wang Z, Zhang X X, Huang K, Miao Y, Shi P, Liu B, Long C, Li A (2013). Metagenomic profiling of antibiotic resistance genes and mobile genetic elements in a tannery wastewater treatment plant. PLoS One, 8(10): e76079

Xiao K Q, Li B, Ma L, Bao P, Xue Zhou X, Zhang T, Zhu Y G (2016). Metagenomic profiles of antibiotic resistance genes in paddy soils from South China. FEMS Microbiology Ecology, 92: fiw023

Yang Y, Li B, Ju F, Zhang T (2013). Exploring variation of antibiotic resistance genes in activated sludge over a four-year period through a metagenomic approach. Environmental Science \& Technology, 47(18): 10197-10205 